\title{
Salivary antioxidants and periodontal disease status
}

\author{
Dean V. Sculley ${ }^{1 *}$ and Simon C. Langley-Evans ${ }^{2}$ \\ ${ }^{I}$ Centre for Healthcare Education, University College Northampton, Boughton Green Road, Northampton NN2 7AL, UK \\ ${ }^{2}$ Division of Nutritional Biochemistry, University of Nottingham, Sutton Bonington Campus, Loughborough LE12 5RD, UK
}

\begin{abstract}
Periodontal disease is a common chronic adult condition. The bacterium Porphyromonas gingivalis has been implicated in the aetiology of this disease, which causes destruction of the connective tissue and bone around the root area of the tooth. It has been observed that invading $P$. gingivalis bacteria trigger the release of cytokines such as interleukin 8 and tumour necrosis factor $\alpha$, leading to elevated numbers and activity of polymorphonucleocytes (PMN). As a result of stimulation by bacterial antigens, PMN produce the reactive oxygen species (ROS) superoxide via the respiratory burst as part of the host response to infection. Patients with periodontal disease display increased PMN number and activity. It has been suggested that this proliferation results in a high degree of ROS release, culminating in heightened oxidative damage to gingival tissue, periodontal ligament and alveolar bone. Antioxidant constituents in plasma have been welldocumented, being chiefly ascorbate, albumin and urate, and these are known to display sensitivity to dietary antioxidant intakes. The concentration of antioxidants in saliva does not appear to mirror those of plasma. The extent of dietary influence upon salivary antioxidant status is unclear. Urate is the predominant salivary antioxidant, with albumin and ascorbate providing minor contributions. Previous research has found reduced salivary antioxidant activity in patients suffering from periodontal disease. An improved understanding of the role antioxidants play in periodontitis, and the influence of nutrition on antioxidant status, may lead to a possible nutritional strategy for the treatment of periodontal disease.
\end{abstract}

Periodontitis: Reactive oxygen species: Antioxidants: Saliva

\section{Porphyromonas gingivalis and oral health}

Periodontal disease is one of the most commonly reported chronic adult conditions. The disease state ranges from gingivitis to periodontitis and advanced periodontitis. Gingivitis is characterised by inflammation of the gum caused by plaque deposits, with possible bleeding when brushed or probed. Gingivitis has a high incidence rate, affecting $50 \%$ of the population (Ridgeway, 2000). Periodontitis can be identified by the hardening of plaque to form calculus, causing gum recession. This results in the formation of pockets between 3.5 and $5.5 \mathrm{~mm}$ between the tooth surface and the gum, and occurs in $80 \%$ of the American population and $51 \%$ of the UK population at some point during their lifetime (Ridgeway, 2000; Nuttall et al. 2001). Advanced periodontitis is distinguished by excessive tissue loss of the gingiva and alveolar bone and pockets greater than $5.5 \mathrm{~mm}$ in depth. This condition often leads to tooth exfoliation due to the destruction of the tooth connective ligaments (Chapple et al. 1997).
The aetiology of periodontal disease is believed to be an imbalance in the bacterial species that colonise the oral cavity, and the host immunological response to these bacterial pathogens (Lamont \& Jenkinson, 1998). There are more than 300 distinct species of bacteria present in the gingival area of the mouth, most of which exist in a commensal relationship with the host. However, three species have been identified as being ubiquitous in periodontal plaque formations. These bacteria, known as periodontal pathogens, are Actinobacillus actinomycetemcomitans, Bacteroides forsythus and Porphyromonas gingivalis (Jenkinson \& Dymock, 1999). Of these, $P$. gingivalis shows extensive proliferation in diseased individuals (Haffajee \& Socransky, 1994; Chapple et al. 1997; Lamont \& Jenkinson, 1998).

$P$. gingivalis is a gram-negative anaerobe that is transmitted via saliva or other vectors from infected individuals (Greenstein \& Lamster, 1997). Antecedent bacteria, such as oral streptococci, are usually required to provide the ideal 
conditions for $P$. gingivalis colonisation, such as growth substrates, reduced $\mathrm{O}_{2}$ tension and as attachment sites themselves. For these reasons, $P$. gingivalis is often involved in secondary colonisation. Major colonisation sites include the teeth, gingiva, cheek and tongue (Theilade, 1990). The mechanisms used for binding include the utilisation of membrane proteins and fimbriae. $P$. gingivalis possesses more than eight haemagglutinins that mediate its binding to host cell receptors, most of which are oligosaccharides (Lamont \& Jenkinson, 2000). The binding to erythrocytes may also provide haem which is required for growth, although $P$. gingivalis also produces proteolytic enzymes that degrade substrates such as collagen, fibronectin, fibrinogen, laminin and keratin, providing molecules necessary for metabolism (Travis et al. 1995; Lamont \& Jenkinson, 2000). Furthermore, bacterial polysaccharides act to counter phagocytosis by host immune cells whilst liposaccharides induce the production of the reactive oxygen species superoxide $\left(\mathrm{O}_{2}{ }^{-}\right)$from polymorphonucleocytes $(\mathrm{PMN})$, resulting in increased inflammation (Jenkinson \& Dymock, 1999).

Fimbriae and proteins assist in the binding to epithelial cells, with the bacterium being taken into the cell cytoplasm but not bound in a vesicle. Once in the cytoplasm, $P$. gingivalis replicates in relative safety from the host immune cells due to its location within the cell. Ingression deeper into connective tissue structures occurs via degradation of the network of cell junctions, leading to a proliferation into the host tissues (Katz et al. 2000). $P$. gingivalis possesses an array of agents that can damage host tissue, modulate host cells and evade detection. Large amounts of the cysteine proteinases Arg-gingipain and Lys-gingipain are produced to facilitate the extraction of peptides and amino acids from the surrounding tissues and in the construction of fimbriae, haemagglutinins and haemoglobin receptor sites (Kadowaki et al. 2000). In addition, phospholipase A production by $P$. gingivalis induces precursors of prostaglandins that may cause prostaglandinmediated bone resorption, whilst alkaline and acid phosphatases contribute to the degradation of alveolar bone (Frank \& Voegel, 1978). Bacterial lipoprotein lipase activates osteoclasts and induces the release of prostaglandin $E_{2}$, interleukin (IL) $1 \beta$ and tumour necrosis factor $\alpha$, all of which mediate bone resorption and reduce osteoblast activity (Havemose-Poulsen \& Holmstrup, 1997). $P$. gingivalis also alters the cellular control of matrix metalloproteinase, thereby disrupting the status of the extracellular matrix and preventing cell repair (Fravalo et al. 1996).

\section{Inflammation responses to Porphyromonas gingivalis}

When stimulated by bacterial pathogens, host cells release pro-inflammatory cytokines as part of the immune response. These include IL- $1 \alpha$ and $-\beta$, IL-6, IL- 8 and tumour necrosis factor $\alpha$. These cytokines recruit PMN to the site of infection (Lamont \& Jenkinson, 1998). PMN play a major role in the aetiology of periodontal disease, as they are the predominant host immune response to oral bacterial infection. Upon stimulation by bacterial antigens, cytokines such as IL-8 promote the PMN to express adhesion molecules and move out of the circulation to the site of infection (Gainet et al. 1998). Here they produce proteolytic enzymes such as elastase, but also $\mathrm{O}_{2} \cdot-$ via the oxidative burst, catalysed by NADPH oxidase. PMN in periodontal patients display an increased number, adhesion and oxidative activity (Asman, 1987). The superoxide is released into the phagosomal enclosure and into the extracellular surroundings (Guarnieri et al. 1991). As the superoxide released is not target-specific, damage to host tissue also occurs. PMN can induce an auto-amplification effect as they can produce IL-8, attracting more PMN into the infection site. This is exacerbated by the ability of $P$. gingivalis to modulate the mobility and function of PMN within the site of infection (Lamont \& Jenkinson, 1998). A reduction of IL- 8 secretion in epithelial cells, mediated by the bacterium, inhibits the recruitment of PMN to the infected area. Their absence results in a build-up of bacteria and an elevation in disease activity (Lamont \& Jenkinson, 2000). P. gingivalis also inhibits PMN chemotaxis by secreting low-molecular-weight molecules such as succinic acid, which is thought to reduce the intracellular $\mathrm{pH}$ of the PMN (Rotstein et al. 1985) and depolarize the PMN membrane causing immobilization (Novak \& Cohen, 1991).

To avoid oxidative destruction by $\mathrm{O}_{2}{ }^{--}$released by PMN, $P$. gingivalis produces the antioxidant superoxide dismutase, converting the $\mathrm{O}_{2}{ }^{--}$to $\mathrm{H}_{2} \mathrm{O}_{2}$. As previously stated, $P$. gingivalis possesses haem, the dominant species of which is u-oxo bishaem of iron protoporphyrin IX produced via ferrous-iron obtained from haemoglobin (Smalley et al. 1998). This may act as a buffer to protect against $\mathrm{H}_{2} \mathrm{O}_{2}$, negating the need for an enzymic degradation system (Smalley et al. 2000). However, $\mathrm{O}_{2}^{--}$and its byproducts $\mathrm{H}_{2} \mathrm{O}_{2}$ and the hydroxyl radical can cause extensive damage to host tissues. This includes depolymerisation of protein constituents, lipid peroxidation and degradation of DNA bases and sugar elements (Greenwald \& Moy, 1979; Halliwell, 1991). Bartold et al. (1984) demonstrated that hydroxyl radicals generated in vitro induced major oxidative damage to isolated porcine gingivae. This injury included depolymerisation of hyaluronic acid and proteoglycans in the extracellular matrix.

One aspect of periodontal disease that is attracting interest is its possible association with cardiovascular diseases such as CHD and cerebrovascular disease. This has been partly attributed to socio-economic factors such as diet, smoking and chronic infections (Fig. 1). Recent research has found that periodontitis and CHD possess similar pathogenic factors and mechanisms. Patients with periodontal disease display increases in the plasma clotting factor fibrinogen and leucocyte count, which is not associated with smoking or social class. This may cause hyperfibrinogeneamia, resulting in an elevated risk of thrombosis development and heart disease (Kinane \& Lowe, 2000). The development of atherosclerosis may be influenced by cytokines produced as a result of oral bacterial infection. Pro-inflammatory cytokines such as IL-1, IL-6 and tumour necrosis factor $\alpha$ produce an increase in adhesion molecule expression on endothelial cells, causing a proliferation of monocyte migration into the subintimal region of arteries and the subsequent development of atherosclerotic plaques (Gamble et al. 1985). These cytokines may also inhibit 


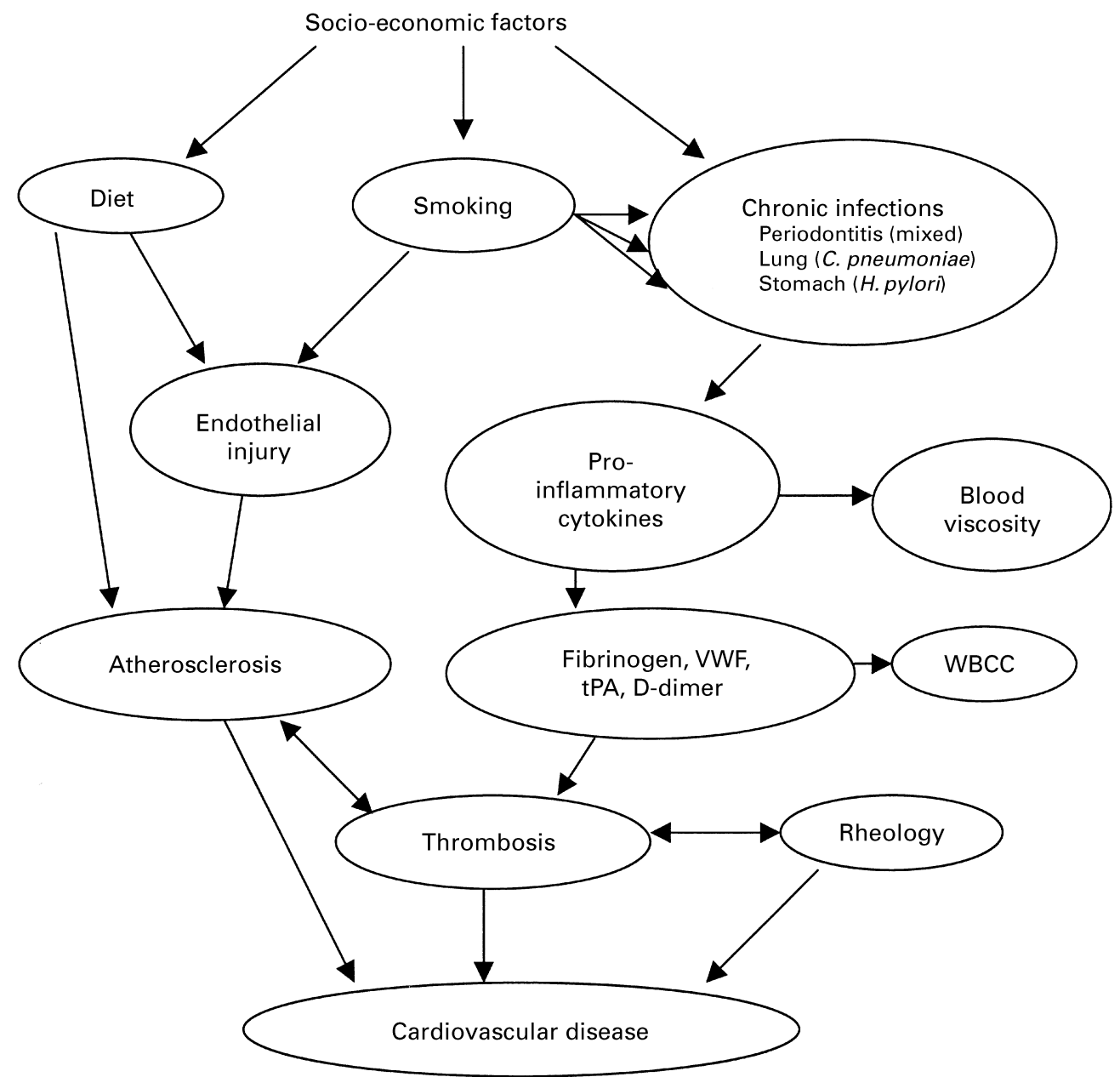

Fig. 1. Cardiovascular risk factors. C. pneumoniae, Chlamydia pneumoniae; H. pylori, Heliobacter pylori; VWF, von Willebrand factor; tPA, tissue plasminogen factor; D-dimer, fibrin D-dimer; WBCC, leucocyte count. (Adapted from Kinane \& Lowe, 2000.)

lipoprotein lipase leading to lipaemia and increased fibrinogen production (Beutler \& Cerami, 1989).

Bacterial lipopolysaccharide from the oral cavity may also be implicated in the pathogenesis of CHD. Under normal conditions, the presence of bacterial lipopolysaccharide in plasma is improbable as it is usually bound to plasma proteins. However, in cases of trauma to the gingiva either by the direct mechanical action during a dental scale or by the chronic presence of an abscess, bacteria may enter the circulation and lead to bacteraemia. Free lipopolysaccharide may cause activation of leucocytes, platelets or the endothelium (Kinane \& Lowe, 2000). The binding of lipopolysaccharide to lipopolysaccharide-binding protein will enable binding to CD14 receptors present on monocytes, macrophages and endothelial surfaces, inducing cellular activation. The resultant increase in adhesion molecule expression by cytokines and chemokines would facilitate leucocyte attachment and migration into the subintimal arterial region causing an increase in the number of foam cells and smooth muscle cells, thereby aiding the formation of atherosclerotic plaque (Tobias et al. 1997). The relationship between periodontal disease and cerebrovascular disease has received little research. In a recent study periodontitis was found to be a major risk factor in cerebrovascular accidents, with particular emphasis on nonhaemorrhage stroke. This is believed to be mediated by the same pro-inflammatory response of the bacteria on cytokines and PMN (Wu et al. 2000). Indeed, bacteria responsible for periodontitis have been found in atherosclerotic plaque deposits of cerebrovascular accident sufferers (Haraszthy et al. 1998).

\section{Salivary antioxidants}

Antioxidants are present in all body fluids and tissues, and protect against endogenously-formed free radicals, usually produced by leakage of the electron transport system (Halliwell, 1991). Antioxidant enzymes such as superoxide dismutase and glutathione peroxidase provide protection within cells whilst low-molecular-weight scavenging antioxidants are present in extracellular fluid. These include ascorbic acid, $\alpha$-tocopherol and $\beta$-carotene. In addition, 
dietary-derived components such as uric acid, non-protein thiols and glutathione also act as antioxidants (Beutler, 1979; Halliwell, 1991), as does albumin found in plasma and saliva (Halliwell, 1988; Moore et al. 1994). Ascorbic acid is believed to be the major aqueous antioxidant, whilst $\alpha$-tocopherol protects against lipid peroxidation (Frei, 1991). The nature and activity of antioxidants in plasma and fluids, such as those lining the epithelial surface of the lungs, have been extensively characterised (Frei, 1991; Abbey, 1995). However, saliva has received less interest and research remains limited.

Whole saliva is a combination of gingival crevicular fluid, which has a composition similar to serum, and fluid released from salivary glands, of which the parotid, submandibular and sublingual are the three major sources (Navazesh, 1993). The components of whole saliva can be seen in Fig. 2. Uric acid appears to be the dominant antioxidant present in saliva and displays a concentration similar to that of serum. Other salivary antioxidants include ascorbic acid and albumin, but concentrations of these are lower than that of serum (Moore et al. 1994). This may indicate an active secretion system for salivary antioxidants rather than passive diffusion from the circulation. This becomes further evident when antioxidant concentrations are compared in unstimulated and stimulated saliva. Stimulated saliva contains a lower concentration of antioxidants but when flow rates are taken into account, antioxidant capacity is higher than in unstimulated saliva (Moore et al. 1994).

There are numerous methods available for saliva collection. These include collection of whole unstimulated saliva, saliva stimulated by using materials such as paraffin wax, gum base or citric acid, or collection of specific gland saliva. When conducting analysis of saliva for antioxidants, whole saliva is more relevant as it contains gingival crevicular fluid, immune cells and tissue metabolites (Navazesh, 1993; Kaufman \& Lamster, 2000). Stimulated saliva has been used in the analysis of antioxidants (Moore et al. 1994). However, as unstimulated flow represents the major intra-oral condition, this would provide a more accurate account of the oral environment and saliva antioxidant composition for analysis (Edgar, 1992). In addition, the stimulation may increase the expulsion of gingival crevicular fluid from the periodontal pocket through the mastication process. This may artificially increase the concentration of antioxidants in the saliva (Chapple et al. 1997).

Previous research considering salivary antioxidant status and periodontal disease is sparse and has yielded conflicting data. This may be a result of the different methodology employed by the authors, though there are other factors that may cause discrepancies. Guarnieri et al. (1991) observed that PMN are found in greater concentration at sites of gingival inflammation. They postulated that $\mathrm{O}_{2}^{--}$produced by PMN as part of the host immune response could cause host tissue oxidative damage if it were not matched by an increase in antioxidant concentration. However, they also speculated that an apparent inhibitory mechanism observed in PMN from periodontal patients may prevent excessive damage to host tissues.

Moore et al. (1994) measured the antioxidant capacity of saliva in diseased and healthy individuals using the Trolox equivalent antioxidant capacity assay. Measurements were made using stimulated and unstimulated saliva samples. No difference was found between the antioxidant capacity of saliva in diseased and healthy patients. However, the diseased sample size was small (seven subjects) and their disease status was not categorized, having been defined only as needing dental treatment. Similarly, definition of the healthy cohort was ambiguous, being described as 'apparently healthy'. Given that $51 \%$ of the UK population (Nuttall et al. 2001) will suffer some degree of gingival disease, it is likely that these controls included some affected individuals. No clinical examination was made on the control group. In addition, saliva samples were stored at $-20^{\circ} \mathrm{C}$; that may have allowed degradation of antioxidant capacity. Chapple et al. (1997) found that antioxidant capacity in serum was $30 \%$ lower in samples stored at $-20^{\circ} \mathrm{C}$ as opposed to under $\mathrm{N}_{2}$ using the Trolox equivalent artioxidant capacity assay.

In a similar study Chapple et al. (1997) studied serum and saliva samples in diseased and control groups using an enhanced chemiluminescent assay. Both groups were well defined, with eighteen periodontal patients and sixteen

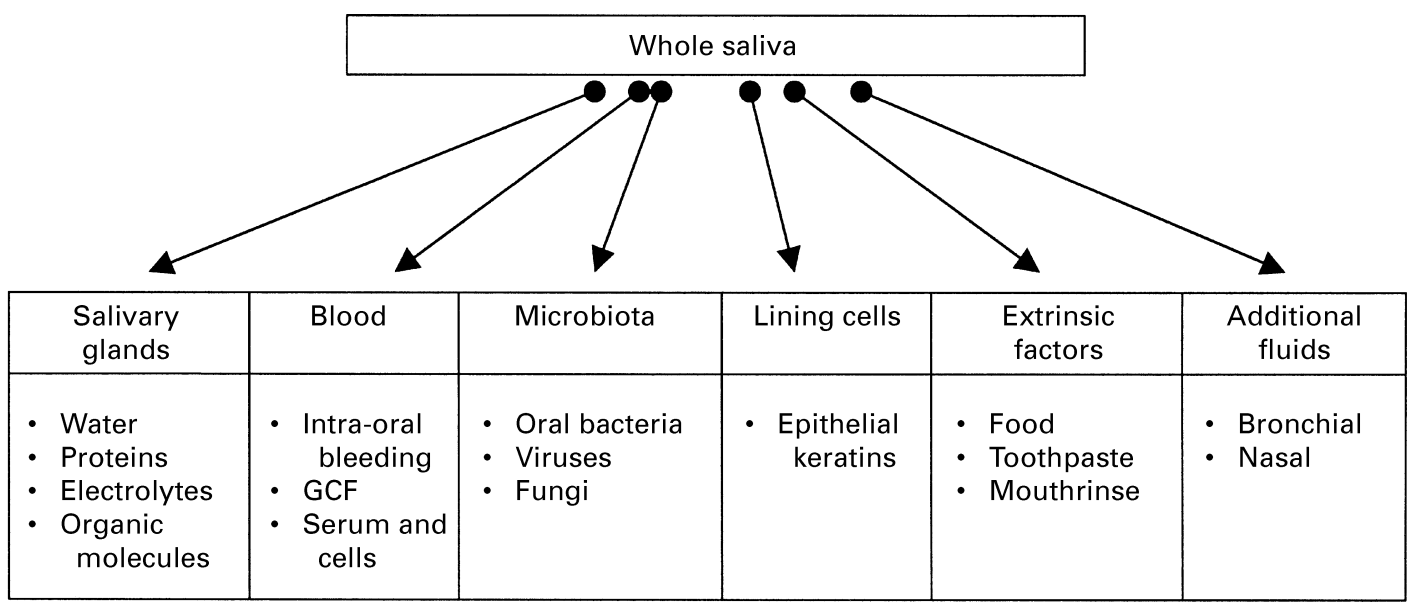

Fig. 2. Components of whole saliva. GCF, gingival crevicular fluid. (Adapted from Kaufman \& Lamster, 2000.) 
controls. Serum antioxidant capacity in the two groups was found to be similar. However, saliva antioxidant capacity was significantly lower in diseased patients compared with controls. In addition, saliva antioxidants: serum antioxidants was also significantly lower in the diseased patients. It was proposed that the reduction in antioxidant capacity was either a direct causal factor in the periodontal disease patients, or that the reduction was due to a reduction in scavenging antioxidants mediated through an increase in oxidative stress.

Clearly, there is a need for standardisation of methods for defining periodontal disease severity and saliva sampling and testing protocol. We have used a method for scoring the extent of periodontal disease that is common amongst British dental practices. It involves the examination of the gingiva with the use of a periodontal probe. This graduated probe is inserted between the tooth and the gingiva to determine the depth of any pocket formation. The dental structure is divided into six areas or sextants. Each sextant is given a score of between 0 and 4 , depending on the extent of damage to the gingiva (Table 1). Once the scores for all sextants are collected, the sum of these scores is subtracted from 24 to give a rating of periodontal disease. A score of 0 would indicate extensive disease in all sextants, whilst healthy gingiva with no evidence of disease would score 24 . This system provides a quick and convenient method of assessing periodontal disease severity in individuals, whilst indicating the location in the dental structure of the disease.

The method used for saliva collection and storage will influence the antioxidant capacity upon analysis. We have employed a collection protocol utilising 'salivette' tubes, but using unstimulated saliva. The saliva is collected with the individual seated. Saliva is allowed to pool in the bottom of the mouth and collected into a plastic vial. Upon completion of the timed collection, the saliva volume is measured and then inserted into a salivette tube (natural cotton insert) and centrifuged at $3000 \mathrm{rpm}$ at $4^{\circ} \mathrm{C}$ for $5 \mathrm{~min}$. The supernatant fraction is then stored at $-80^{\circ} \mathrm{C}$ until analysed. This process provides a saliva sample free of large particle debris and of reduced viscosity, allowing more accurate and reproducible analysis.

Our proposed study will investigate the relationship between salivary antioxidant status and periodontal disease. A group of 200 non-smoking adults attending for routine check-up examination will be recruited. Patients taking nutritional supplements will be excluded from the study.

Table 1. Scoring index for periodontal examination (adapted from Ainamo et al. 1982)

\begin{tabular}{ll}
\hline Score & Description of gingiva \\
\hline 0 & $\begin{array}{c}\text { No bleeding plaque-retention factors or pocketing } \\
>3.5 \mathrm{~mm} .\end{array}$ \\
1 & $\begin{array}{c}\text { Bleeding on probing but no plaque-retentive factors or } \\
\text { pocketing }>3.5 \mathrm{~mm} .\end{array}$ \\
& $\begin{array}{c}\text { Plaque-retentive factors present but no pocketing } \\
>3.5 \mathrm{~mm} .\end{array}$ \\
3 & Pockets $>3.5 \mathrm{~mm}$ but $<5.5 \mathrm{~mm}$. \\
4 & Pockets $>5.5 \mathrm{~mm}$. \\
\hline
\end{tabular}

During the dental examination, the patient will be assigned a periodontal disease score. Saliva will be collected and analysed for total antioxidant capacity and individual antioxidants. In addition, neutrophil numbers will be calculated and prostaglandin $\mathrm{E}_{2}$ will be estimated using ELISA.

In a further two proposed studies we will investigate the relationship between antioxidants in saliva and serum. We will recruit two groups of twenty volunteers, one group of smokers and one of healthy controls. Fasting saliva and blood samples will be taken, with analysis of total and individual antioxidants calculated in both samples. We will investigate the influence of antioxidant intake on salivary and serum antioxidants levels. A 4-week programme of antioxidant vitamin and mineral supplementation (Se, and vitamins $\mathrm{A}, \mathrm{C}$ and $\mathrm{E}$ ) will follow baseline saliva and serum antioxidant calculations in twenty non-smoking volunteers. In addition to the prescribed antioxidant intake, subjects will be required to complete a weighed food record for analysis. Saliva and serum samples will be taken mid-way through the study and at the end of the 4-week supplementation period.

Preliminary data obtained from a total of eighteen dental patients indicates a non-significant ' $U$ ' relationship between the antioxidant capacity measured using the ferric reducing ability of plasma assay (Benzie \& Strain, 1996) and the periodontal score (Fig. 3). The high antioxidant value for individuals with the most advanced stages of disease has not been found in previous research (Moore et al. 1994; Chapple et al. 1997). This may be because individuals used in these studies did not have severe periodontitis. Another factor may be leakage of gingival crevicular fluid into saliva, which is more pronounced in the larger periodontal pockets associated with severe periodontal disease (Chapple et al. 1997). A third possibility is that there may be an active secretion of antioxidants into the gingival area in response to

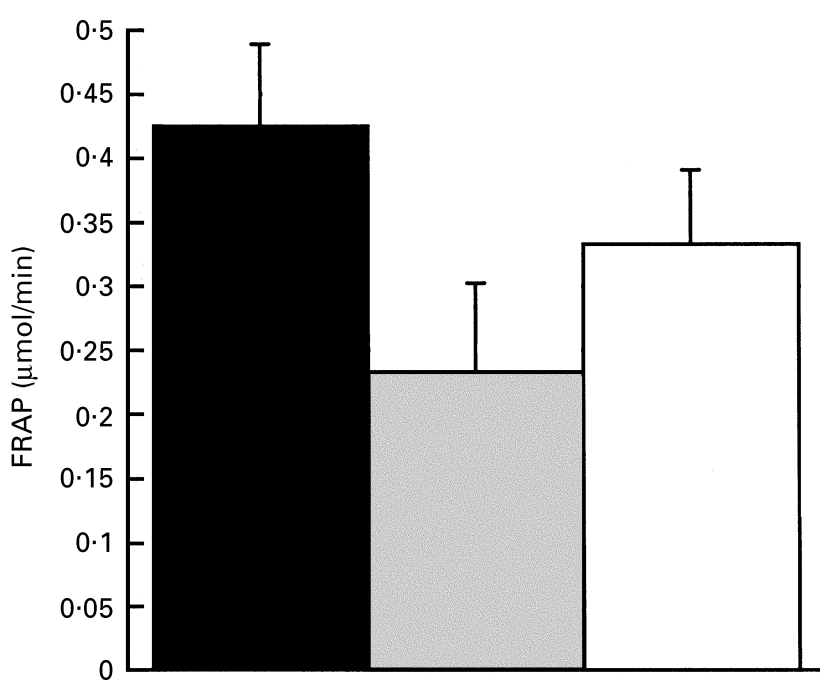

Fig. 3. Relationship between antioxidant capacity (ferric reducing ability of plasma; FRAP) and periodontal score. ( $\boldsymbol{\square})$, Periodontal score of 1-12, extensive disease; ( $\square$ ), periodontal score of 13-20, moderate disease; $(\square)$, periodontal score of $21-24$, little or no disease. Values are means with their standard errors represented by vertical bars. For details of procedures, see Table 1 and p.140. 
extensive oxidative damage. Further study is needed to clarify these findings.

Periodontal disease is clearly an important and potentially life-threatening condition, often underestimated by health professionals and the general population. The available evidence implicating inflammatory mediators and cells in the disease process suggests that local antioxidant status may be of importance in determining susceptibility to the disease and its progression following initial bacterial colonisation. It is now of importance to determine the possible contribution of diet to salivary antioxidant status. In the future antioxidant supplementation may be used in the treatment or prevention of these chronic diseases of the oral cavity.

\section{References}

Abbey M (1995) The importance of vitamin-E in reducing cardiovascular risk. Nutrition Reviews 53, S28-S32.

Ainamo J, Barmes D, Beagrie G, Cutress T, Martin J \& Sardo-infiri J (1982) Development of the World Health Organization community periodontal index of treatment needs (CPITN). International Dental Journal 32, 281-291.

Asman B (1987) Peripheral PMN cells in juvenile periodontitis. Journal of Clinical Periodontitis 15, 360-364.

Bartold PM, Wiebkin OW \& Thonard JC (1984) The effect of oxygen-derived free radicals on gingival proteoglycans and hyaluronic acid. Journal of Periodontal Research 19, 390-400.

Benzie IFF \& Strain JJ (1996) The ferric reducing ability of plasma (FRAP) as a measure of "antioxidant power": The FRAP assay. Analytical Biochemistry 239, 70-76.

Beutler E (1979) Glutathione peroxidase. In Red Cell Metabolism: A Manual of Biochemical Methods, 2nd ed., pp. 71-73 [E Beutler, editor]. New York: Grune and Stratton.

Beutler BA \& Cerami A (1989) Recombinant interleukin-1 suppresses lipoprotein lipase activity in 3T3-L1 cells. Journal of Immunology 135, 3969-3971.

Chapple ILC, Mason GI, Garner I, Matthews JB, Thorpe GH, Maxwell SRJ \& Whitehead TP (1997) Enhanced chemiluminescent assay for measuring the total antioxidant capacity of serum, saliva and crevicular fluid. Annals of Clinical Biochemistry 34, 412-421.

Edgar WM (1992) Saliva: Its secretion, composition and functions. British Dental Journal 172, 305-312.

Frank RM \& Voegel JC (1978) Bacterial bone resorption in advanced cases of human periodontitis. Journal of Periodontal Research 13, 251-261.

Fravalo P, Menard C \& Bonnaure-Mallet M (1996) Effect of Porphyromonas gingivalis on epithelial cell MMP-9 type IV collagenase production. Infection and Immunity 64, 4940-4945.

Frei B (1991) Ascorbic acid protects lipids in human plasma and low density lipoprotein against oxidative damage. American Journal of Clinical Nutrition 54, 1113S-1118S.

Gainet J, Chollet-Martin S, Brion M, Hakim J, Gougerot-Pocidalo M \& Elbim C (1998) Interleukin-8 production by polymorphonuclear neutrophils in patients with rapidly progressive periodontitis: An amplifying loop of polymorphonuclear neutrophil activation. Laboratory Investigation 78, 755-761.

Gamble JR, Harlan JM \& Klebanoff SJ (1985) Stimulation of the adherence of neutrophils to umbilical vein endothelium by human recombinant tumor necrosis factor. Proceedings of the National Academy of Sciences USA 82, 8667-8671.

Greenstein G \& Lamster I (1997) Bacterial transmission in periodontal diseases: a critical review. Journal of Periodontology 68 , 421-431.
Greenwald RA \& Moy WW (1979) Inhibition of collagen gelation by action of the superoxide radical. Arthritis and Rheumatism 22, 251-259.

Guarnieri C, Zucchelli G, Bernardi F, Scheda M, Valentini AF \& Calandriello M (1991) Enhanced superoxide production with no change of the antioxidant activity in gingival fluid of patients with adult periodontitis. Free Radical Research 15, 11-16.

Haffajee AD \& Socransky SS (1994) Microbial etiological agents of destructive periodontal diseases. Periodontology 5, 78-111.

Halliwell B (1988) Albumin - An important extracellular antioxidant? Biochemical Pharmacology 37, 569-571.

Halliwell B (1991) Reactive oxygen species in living systems: source, biochemistry, and role in human disease. American Journal of Medicine 91, Suppl. 3C, 14-22.

Haraszthy VI, Zambon JJ, Trevisan M, Zeid M \& Genco RJ (1998) Identification of pathogens in atheromatous plaques (abstract). Journal of Dental Research 77B, 273Abstr.

Havemose-Poulsen A \& Holmstrup P (1997) Factors affecting IL-1-mediated collagen metabolism by fibroblasts and the pathogenesis of periodontal disease: a review of the literature. Critical Reviews in Oral Biology and Medicine 8, 217-236.

Jenkinson HF \& Dymock D (1999) The microbiology of periodontal disease. Dental Update 26, 191-197.

Kadowaki T, Nakayama K, Okamoto K, Abe N, Baba A, Shi Y, Ratnayake DB \& Yamamoto K (2000) Porphyromonas gingivalis proteinases as virulence determinants in progression of periodontal disease. Journal of Biochemistry 128, 153-159.

Katz J, Sambandam V, Wu JH, Michalek SM \& Balkovetz DF (2000) Characterisation of Porphyromonas gingivalis-induced degradation of epithelial junction complexes. Infection and Immunity 68, 1441-1449.

Kaufman E \& Lamster IB (2000) Analysis of saliva for periodontal diagnosis. Journal of Clinical Periodontology 27, 453-465.

Kinane DF \& Lowe GDO (2000) How periodontal disease may contribute to cardiovascular disease. Periodontology $2000 \mathbf{2 3}$, 121-126.

Lamont RJ \& Jenkinson LRJ (1998) Life below the gum line: Pathogenic mechanisms of Porphyromonas gingivalis. Microbiology and Molecular Biology Reviews 62, 1244-1263.

Lamont RJ \& Jenkinson LRJ (2000) Subgingival colonization by Porphyromonas gingivalis. Oral Microbiology and Immunology 15, 341-349.

Moore S, Calder KAC, Millar NJ \& Rice-Evans CA (1994) Antioxidant activity of saliva and periodontal disease. Free Radical Research 21, 417-425.

Navazesh M (1993) Methods for collecting saliva. Annals of the New York Academy of Science 694, 72-77.

Novak MJ \& Cohen HJ (1991) Depolarization of polymorphonuclear leukocytes by Porphyromonas (Bacteroides) gingivalis 381 in the absence of respiratory burst activation. Infection and Immunity 59, 3134-3142.

Nuttall NM, Steele JG, Pine CM, White D \& Pitts NB (2001) The impact of oral health on people in the UK in 1998. British Dental Journal 190, 121-126.

Ridgeway EE (2000) Periodontal disease: Diagnosis and management. Journal of the American Academy of Nurse Practitioners 12, 79-83.

Rotstein OD, Pruett TL, Fiegel VD, Nelson RD \& Simmons RL (1985) Succinic acid, a metabolic by-product of Bacteriodes species, inhibits polymorphonuclear leukocyte function. Infection and Immunity 48, 402-408.

Smalley JW, Birss AJ \& Silver J (2000) The periodontal pathogen orphyromonas gingivalis harnesses the chemistry of the u-oxo bishaem of iron protoporphyrin IX to protect against hydrogen peroxide. FEMS Microbiology Letters 183, 159-164.

Smalley JW, Silver J, Marsh PJ \& Birss AJ (1998) The periodontopathogen Porphyromonas gingivalis binds iron 
protoporphyrin IX in the u-oxo dimeric form: an oxidative buffer and possible pathogenic mechanism. Biochemistry Journal 331, 681-685.

Theilade E (1990) Factors controlling the microflora of the healthy mouth. In Human Microbial Ecology, pp. 1-56 [MJ Hill and PD Marsh, editors]. Boca Raton, FL: CRC Press, Inc.

Tobias PS, Gegner J, Tapping R, Orr S, Mathison J, Lee KJD, Kravchenko V, Han J \& Ulevitch RJ (1997) Lipopolysaccharide dependant cellular activation. Journal of Periodontal Disease 32, 99-103.

Travis J, Potempa J \& Maeda H (1995) Are bacterial proteinases pathogenic factors? Trends in Microbiology 3, 405-407.

Wu T, Trevisan M, Genco RJ, Dorn JP, Falkner KL \& Sempos T (2000) Periodontal disease and risk of cerebrovascular disease. Archives of International Medicine 160, 2749-2755. 